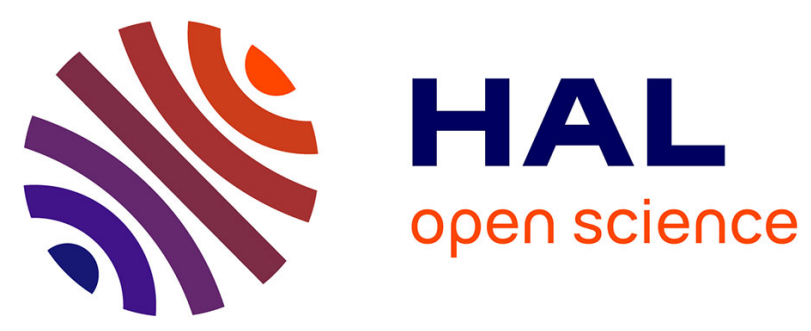

\title{
Hospitalisations et mortalité des jeunes vivant avec une pathologie chronique en France: analyse à partir de l'échantillon généraliste des bénéficiaires
}

Antoine Rachas, Philippe Tuppin, Elise de La Rochebrochard, Albert Faye, Laurence Meyer, Josiane Warszawski

\section{To cite this version:}

Antoine Rachas, Philippe Tuppin, Elise de La Rochebrochard, Albert Faye, Laurence Meyer, et al.. Hospitalisations et mortalité des jeunes vivant avec une pathologie chronique en France: analyse à partir de l'échantillon généraliste des bénéficiaires. Epidemiology and Public Health $=$ Revue d'Epidémiologie et de Santé Publique, 2017, 65 (Suppl. 1), pp.S24-S25. 10.1016/j.respe.2017.01.057 . hal-02422794

\section{HAL Id: hal-02422794 \\ https://hal.science/hal-02422794}

Submitted on 18 Feb 2020

HAL is a multi-disciplinary open access archive for the deposit and dissemination of scientific research documents, whether they are published or not. The documents may come from teaching and research institutions in France or abroad, or from public or private research centers.
L'archive ouverte pluridisciplinaire HAL, est destinée au dépôt et à la diffusion de documents scientifiques de niveau recherche, publiés ou non, émanant des établissements d'enseignement et de recherche français ou étrangers, des laboratoires publics ou privés. 
Rachas Antoine, Tuppin Philippe, La Rochebrochard Elise (de), Faye Albert, Meyer Laurence, Warszawski Josiane, 2017, « Hospitalisations et mortalité des jeunes vivant avec une pathologie chronique en France : analyse à partir de l'échantillon généraliste des bénéficiaires », Revue d'Epidémiologie et de Santé Publique, 65, Suppl. 1, p. S24-S25. DOI: $10.1016 /$ j.respe.2017.01.057.

\section{Hospitalisations et mortalité des jeunes vivant} avec une pathologie chronique en France : analyse à partir de l'échantillon généraliste des bénéficiaires

Antoine Rachas ${ }^{a, b, *}$, Philippe Tuppin ${ }^{c}$, Elise de la Rochebrochard ${ }^{a, d}$, Albert Faye ${ }^{\text {e,f,g }}$, Laurence Meyer ${ }^{\text {a,b }}$, Josiane Warszawski a,b

a Université Paris-Saclay, université Paris-Sud, UVSQ, CESP, Inserm, Villejuif, France

${ }^{b}$ AP-HP, groupe hospitalier Paris-Sud, service d'épidémiologie et santé publique, Le Kremlin-Bicêtre, France

c Caisse nationale d'assurance maladie des travailleurs salariés, direction de la stratégie, des études et des statistiques, département d'études sur les pathologies et les patients, Paris, France

d Institut national d'études démographiques (Ined,), Paris, France

e Université Denis Diderot, Sorbonne Paris Cité, Paris, France

${ }^{f}$ Pédiatrie générale, hôpital Robert Debré, AP-HP, Paris, France

g Inserm U1123, Paris, France

* Auteur correspondant.

Adresse e-mail : antoine.rachas@gmail.com (A. Rachas)

Introduction. Avec l'amélioration du pronostic des pathologies chroniques à début pédiatrique, un nombre grandissant de patients atteignent l'âge adulte. Cependant, l'adolescence et l'entrée dans la vie adulte s'accompagnent de multiples changements et de crises, pouvant entraîner des ruptures avec le système de soins et des complications médicales. Nous avons estimé la prévalence des pathologies chroniques graves à 14 ans puis comparé les incidences des hospitalisations et des décès avec celles de la population générale. 
Méthodes. Une cohorte rétrospective de jeunes ayant atteint 14 ans entre 2005 et 2013, issue de l'échantillon généraliste des bénéficiaires, a permis d'estimer la prévalence des antécédents de déclaration en affection de longue durée (ALD) avant 14 ans, les taux de mortalité, et les probabilités d'hospitalisation en court séjour avant 19 ans (méthode de Kaplan-Meier). L'évolution de l'incidence des hospitalisations par âge a été décrite entre 14 et 21 ans.

Résultats. Parmi 54098 sujets de 14 ans, 3,2 \% (intervalle de confiance à 95\%:3,0-3,3, $n=1811$ ) avaient un antécédent d'ALD. Le taux demortalité pour 10000 personnes-années entre 14 et 19 ans était de $21,2(13,0-34,6)$ chez les sujets ayant une ALD et $1,6(1,2-2,2)$ chez les sujets sans ALD, avec une surmortalité masculine chez les sujets sans ALD uniquement. Les probabilités d'hospitalisation à cinq ans étaient de $56,4 \%$ et $36,6 \%$, respectivement, et $20,8 \%$ et $11,6 \%$ pour les hospitalisations par les urgences. Entre 14 et 21 ans, l'incidence des hospitalisations augmentait chez les jeunes sans ALD, mais chutait à 19 ans chez ceux ayant une ALD. En revanche, l'incidence des hospitalisations par les urgences ne diminuait pas.

Discussion/conclusion. Des études investiguant les stratégies d'évitement des situations à risque permettraient de mieux comprendre l'absence de surmortalité masculine chez les malades. La chute de l'incidence des hospitalisations à 19 ans peut refléter l'atténuation de certaines pathologies avec l'âge, mais aussi des modifications de prise en charge lors du transfert en service d'adultes (discontinuité du suivi, soins préférentiellement ambulatoires ...).

Mots clés. Pathologies chroniques ; Adolescents ; Échantillon généraliste des bénéficiaires

Déclaration de liens d'intérêts. Les auteurs déclarent ne pas avoir de liens d'intérêts. 